\title{
MATHEMATICAL DESCRIPTION OF THE BENDING LiNe OF THE MOHR's LiMITING STRESS CiRCleS
}

\author{
DUISHENALIEV, T., DUISHEMBIEV, A., \\ MERKURYEV, I. \& USHANOV, S.
}

Abstract: The main task of Mohr's theory of strength is a quantitative description of destructive stress states under conditions of triaxial stress states. However, Mohr's theory of strength has not yet had a single quantitative expression. As such an expression, many relationships were proposed, which were put forward based on various considerations. However, any proposed regularity is of little value, unless its quantitative description is established. Moreover, attempts of quantitative description can lead to confusion, the emergence of many perceptions and points of view explaining the same phenomenon. A new equation of the envelope line of limit stress circles is obtained. It differs from all previously known equations in that it has a rigorous mathematical foundation.

Key words: stress circles, envelope line, envelope equation, Mohr's theory of strength.
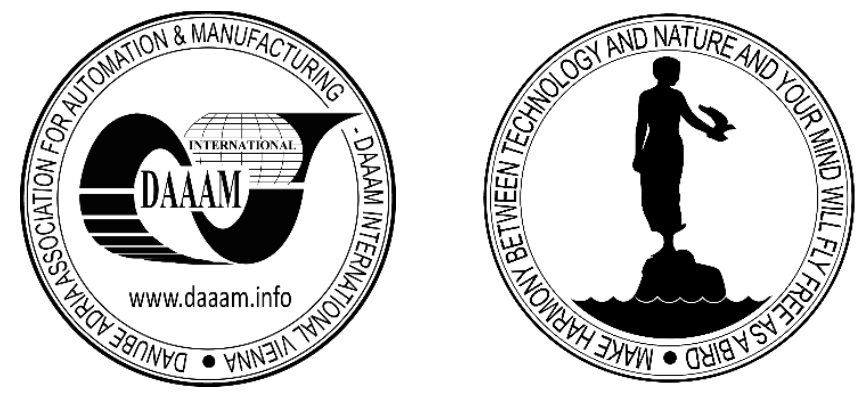

Authors' data: Doctor of Physics and Mathematical Sciences, Professor Duishenaliev, T[uratbek]*; Post-graduate Student Duishembiev, A[lmaz]**, Doctor of Technical Sciences, Professor Merkuryev, I[gor]*, Student Ushanov, S[ergey]*, *National Research University "Moscow Power Engineering Institute" Krasnokazarmennaya 17, Moscow, 111250 Russia, duyshenaliyevt@mpei.ru, **Kyrgyz State Technical University named after I.Razzakov, Ch.Aitmatov Ave. 66, Bishkek, Kyrgyzstan, duishembiev.almaz@gmail.com

This Publication has to be referred as: Duishenaliev, T[uratbek]; Duishembiev, A[lmaz]; Merkuryev, I[gor] \& Ushanov, S[ergey] (2021). Mathematical Description of the Bending Line of the Mohr's Limiting Stress Circles, Chapter 08 in DAAAM International Scientific Book 2021, pp.095-106, B. Katalinic (Ed.), Published by DAAAM International, ISBN 978-3-902734-31-0, ISSN 1726-9687, Vienna, Austria DOI: $10.2507 /$ daaam.scibook.2021.08 


\section{Introduction}

Experimental researches confirmed the consistency of O. Mohr's hypothesis that the largest stress circles, corresponding to the moment of material destruction, form a regular family. Here, by regularity, we mean the fact that these circles are located in such a way that an envelope line can be drawn to them

$$
\tau=f(\sigma) \text {. }
$$

But what is this line, i.e. what is the function $f(\sigma)$ ? There is still no satisfactory answer to this question. Very briefly, the essence of the problem can be expressed as follows. The regularity suggested by Mohr exists, but there is no quantitative expression of it. In other words, there is an envelope line, but there is no generally accepted equation for it. In this case, the problem is far from being solved. The numerous representations of this description can be divided into two groups:

- representations in the form of known lines (straight line, parabola, hyperbola, etc.) (Duishenaliev, 2017);

- representations in the form of given equations (equations of Protodyakonov M.M., Stavrogin A.N., etc.) (Protodiakonov, 1962; Stavrogin, 1961).

But they all correctly describe the behavior of a body only near those stress states that were used to determine the constants of materials. With distancing from these stress states, the deviations increase more and more (Duishenaliev, 2017; Karman, 1915; Koifman et al. 1964; Kuntysh, 1964; Mohr, 1915; Protodiakonov, 1962; Stavrogin, 1961). Such is the state of the theory, which has so many types of its quantitative description. Today there is no single generally accepted quantitative description of this theory.

Establishing the equation of the envelope line is of great practical importance. Rock mass is in triaxial compression. The degree of such compression is different at different depths of the earth's crust. Under what stress conditions is this or that rock destroyed?

Structural and building materials are also in different stress states depending on the applied loads (Duishenaliev et al., 2018). What stress states are destructive for them? The answer to this question is of paramount importance in mining and designing various structures. In light of this, the mathematical description of the envelope line of O. Mohr's limit circles acquired independent scientific and practical significance.

\section{Normal and shear stresses. Limiting stress circles}

The stress state at any point of this body is described by a three-dimensional tensor:

$$
\sigma_{i j}=\left(\begin{array}{lll}
\sigma_{11} & \sigma_{12} & \sigma_{13} \\
\sigma_{21} & \sigma_{22} & \sigma_{23} \\
\sigma_{31} & \sigma_{32} & \sigma_{33}
\end{array}\right),
$$


where $\sigma_{i j}$-stress tensor components. The stress vector on the site with an external normal $n n=n_{i} e_{i}$ has the form:

$$
\sigma_{n}=\sigma_{j i} n_{j} e_{i}
$$

Here $e_{i}$ - unit vectors of a rectangular Cartesian coordinate system. This vector in an expanded form:

$$
\sigma_{n}=\sum_{i=1}^{3} \sum_{j=1}^{3} \sigma_{j i} n_{j} e_{i}
$$

The square of the modulus of this vector:

$$
\sigma_{n}^{2}=\sigma_{n} \cdot \sigma_{n}=\sigma_{j i} n_{j} e_{i} \sigma_{k p} n_{k} e_{p}=\sigma_{j i} \sigma_{k i} n_{j} n_{k}
$$

In expanded form, this expression has the form:

$$
\sigma_{n}^{2}=\sum_{i=1}^{3} \sum_{j=1}^{3} \sum_{k=1}^{3} \sigma_{j i} \sigma_{k i} n_{j} n_{k} .
$$

Projecting vector (2) on the direction of the normal, we find its normal component:

$$
\sigma=\sigma_{i j} n_{i} n_{j}
$$

or in expanded record:

$$
\sigma=\sum_{i=1}^{3} \sum_{j=1}^{3} \sigma_{i j} n_{i} n_{j}
$$

We find the tangential stress from the expression:

$$
\tau= \pm \sqrt{\sigma_{n}^{2}-\sigma^{2}}
$$

Let's substitute here the values $\sigma_{n}, \sigma$ :

$$
\tau= \pm \sqrt{\sigma_{j i} \sigma_{k i} n_{j} n_{k}-\left(\sigma_{j i} n_{j} n_{i}\right)^{2}} .
$$

Expanded:

$$
\tau= \pm \sqrt{\sum_{i=1}^{3} \sum_{j=1}^{3} \sum_{k=1}^{3} \sigma_{j i} \sigma_{k i} n_{j} n_{k}-\left(\sum_{i}^{3} \sum_{j}^{3} \sigma_{j i} n_{j} n_{i}\right)^{2}} .
$$

If the stress components $\sigma_{i j}$ are known, then using formulas (4), (5) it is possible to determine the values of the normal and shear stress at any site. Hence it follows that stresses at any site can be depicted as a point on a plane $\sigma, \tau$. In these expressions $\sigma_{i j}$ 
Duishenaliev, T.; Duishembiev, A.; Merkuryev, I. \& Ushanov, S.: Mathematical De...

is known, and the direction cosines of the normal $n_{i}$ to the planes on which the normal $\sigma$ and shear $\tau$ stresses are calculated are set by ourselves. Expressions (4), (5) are greatly simplified if we go to the main axes. Let's denote the principal stresses through $\sigma_{1} \geq \sigma_{2} \geq \sigma_{3}$, assuming the compressive stresses to be positive values. In this case, the stress state is depicted in the form of points enclosed between three circles and located on these circles themselves. The equations of these circles are as follows:

$$
\begin{aligned}
& \left(\frac{\sigma_{1}+\sigma_{2}}{2}-\sigma\right)^{2}+\tau^{2}=\left(\frac{\sigma_{1}-\sigma_{2}}{2}\right)^{2} \\
& \left(\frac{\sigma_{2}+\sigma_{3}}{2}-\sigma\right)^{2}+\tau^{2}=\left(\frac{\sigma_{2}-\sigma_{3}}{2}\right)^{2} . \\
& \left(\frac{\sigma_{1}+\sigma_{3}}{2}-\sigma\right)^{2}+\tau^{2}=\left(\frac{\sigma_{1}-\sigma_{3}}{2}\right)^{2} .
\end{aligned}
$$

We write the equations of the points of these circles in the form of functions of normal stress:

$$
\begin{aligned}
& \tau(\sigma)= \pm \sqrt{-\left(\sigma-\sigma_{1}\right)\left(\sigma-\sigma_{2}\right)}, \\
& \tau(\sigma)= \pm \sqrt{-\left(\sigma-\sigma_{2}\right)\left(\sigma-\sigma_{3}\right)} \\
& \tau(\sigma)= \pm \sqrt{-\left(\sigma-\sigma_{3}\right)\left(\sigma-\sigma_{1}\right)} .
\end{aligned}
$$

Fig. 1 shows the locations of these circles.

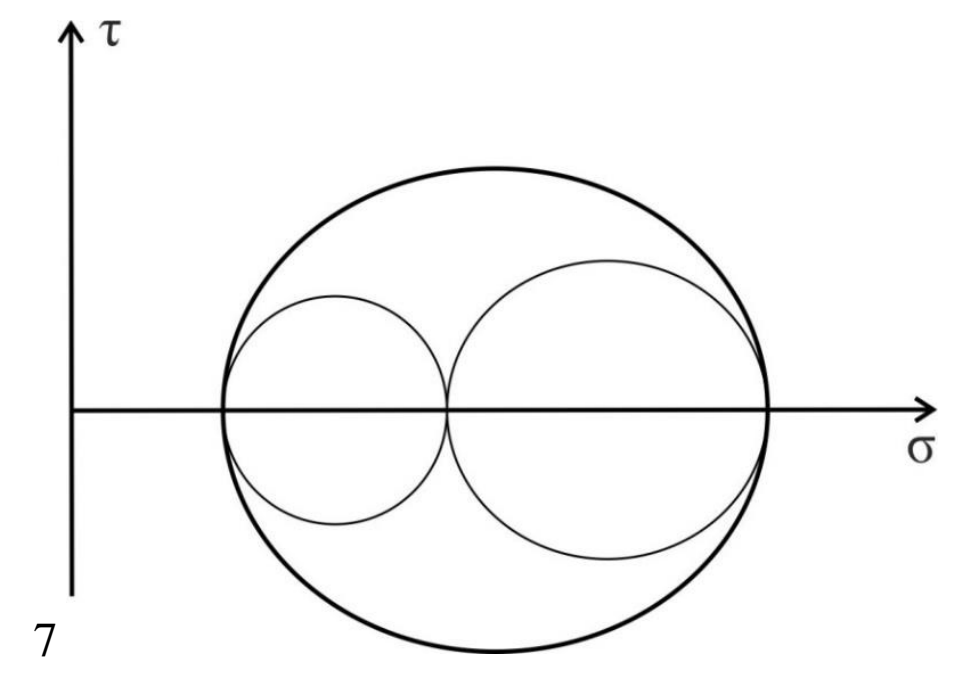

Fig. 1. Image of the stress state on the plane of normal and shear stresses 
In the previous expressions, the value of the normal stress $\sigma$ varies within:

$$
\sigma_{3} \leq \sigma \leq \sigma_{1}
$$

At the same values of the normal stress $\sigma$, the maximum shear stress $\tau$ in absolute value is determined by the expression (11). The circle defined by this expression is called the largest stress circle. The circles are called limiting:

$$
\left(\frac{\sigma_{1}+\sigma_{3}}{2}-\sigma\right)^{2}+\tau^{2}=\left(\frac{\sigma_{1}-\sigma_{3}}{2}\right)^{2}
$$

where the main stresses $\sigma_{1}, \sigma_{3}$ correspond to the moment of the onset of destruction. Here and below, only limiting stress states are considered, i.e. states in which the material is destroyed.

\section{Derivation of the envelope line equation}

The work (Duishenaliev, 2017) provides an overview and analysis of the most used types of the envelope line. It should be noted that these types are not derived, but given. For example, it is first suggested that the envelope line is cycloidal, and then the parameters of the cycloid are determined. Is it possible to do otherwise? Not to set, but to define the function of the envelope line.

The envelope line has one peculiarity. This feature of the envelope line is that it flattens and becomes parallel to the normal stress axis at large values of the principal stresses, as shown on the Figure 2.

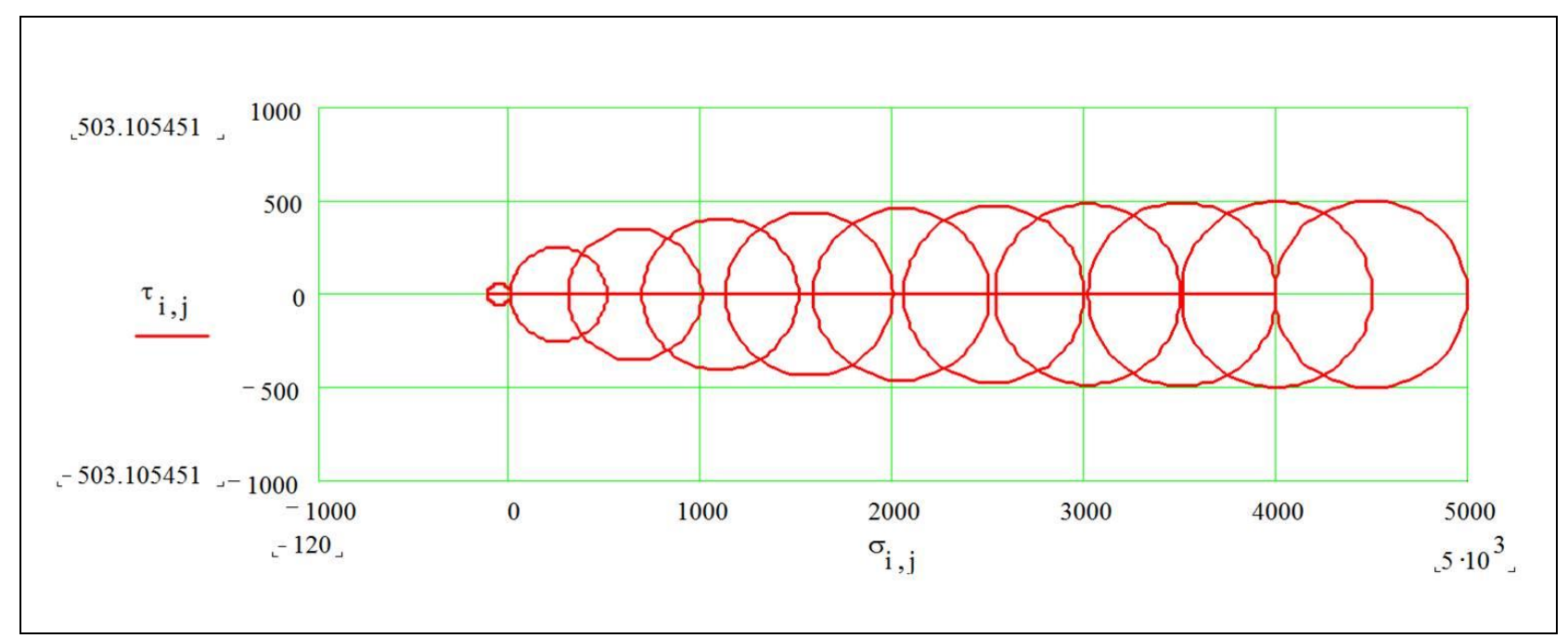

Fig. 2. Regular family of Mohr limit circles

In this area, the radii of the limiting circles become constant:

$$
\left(\sigma_{1}-\sigma_{3}\right) / 2=\text { const }
$$


Duishenaliev, T.; Duishembiev, A.; Merkuryev, I. \& Ushanov, S.: Mathematical De...

By differentiating this equality with respect to the argument $\sigma_{1}$, we find:

$$
\sigma_{3}^{\prime}=\frac{d \sigma_{3}}{d \sigma_{1}}=1
$$

It should be borne in mind that the equality (15) is valid only in the area of high pressures, where the envelope line becomes parallel to the normal stress axis. Is it possible to derive the equation of the envelope line using the expression (15)? Let's try to do this.

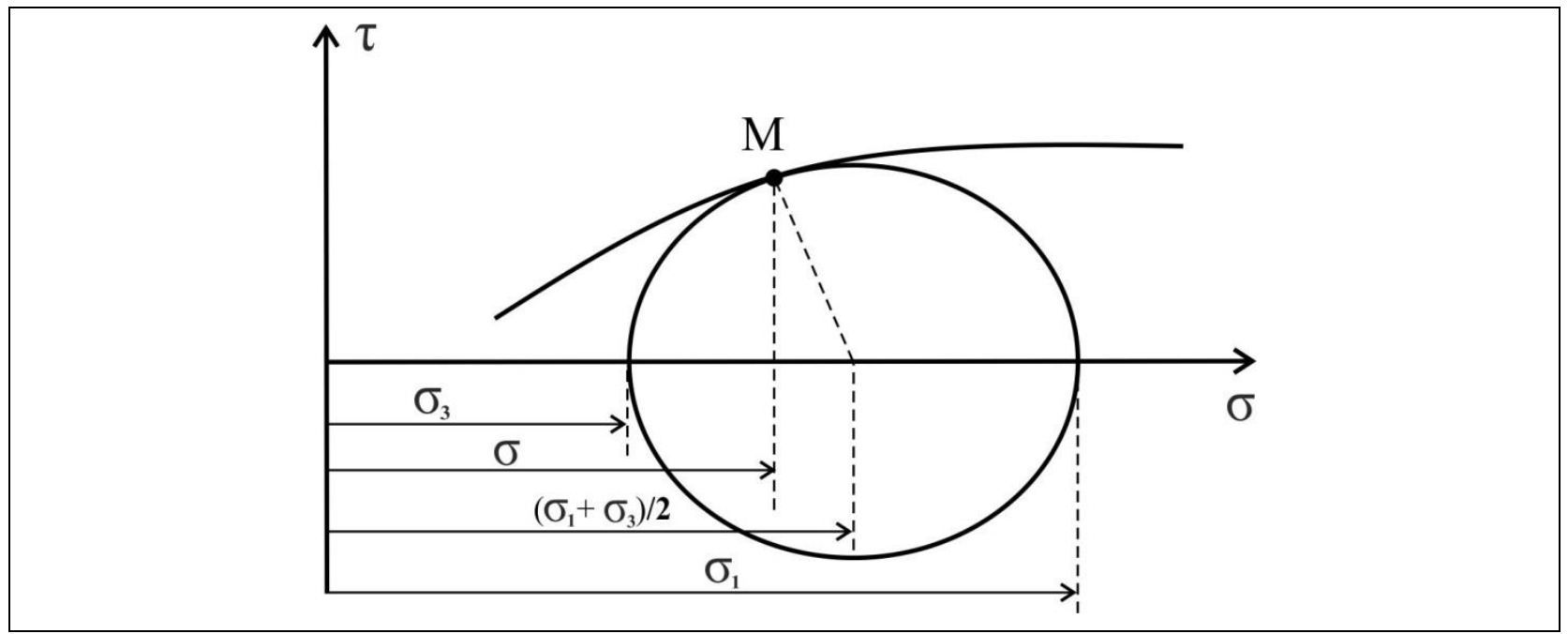

Fig. 3. Mohr's envelope line and limit circle

Let's consider one of the limiting stress circles (Fig. 3). Let at point $\mathrm{M}$ this circle touches the envelope line. The coordinates of this point will be denoted by $\sigma, \tau$. The limiting circle equation has the form (Korn, 1968):

$$
\left(\frac{\sigma_{1}+\sigma_{3}}{2}-\sigma\right)^{2}+\tau^{2}=\left(\frac{\sigma_{1}-\sigma_{3}}{2}\right)^{2}
$$

where $\sigma_{1}, \sigma_{3}$ - the biggest and the smallest principal stresses of the limiting state. We write this equation in the form:

$$
\sigma^{2}+\tau^{2}-\left(\sigma_{1}+\sigma_{3}\right) \sigma+\sigma_{1} \sigma_{3}=0
$$

The family of circles (16) must have an envelope line. In view of this, this family must depend on one parameter. We take the principal stress $\sigma_{1}$ as such a parameter. For the presence of an envelope line, it is necessary that the partial derivative of the equation (16) with respect to this parameter equal to zero:

$$
-\left(1+\sigma_{3}^{\prime}\right) \sigma+\left(\sigma_{3}+\sigma_{1} \sigma_{3}^{\prime}\right)=0 \text {. }
$$


From here we find:

$$
\sigma=\frac{\sigma_{3}+\sigma_{1} \sigma_{3}^{\prime}}{1+\sigma_{3}^{\prime}}
$$

Substitute (17) into (16):

$$
\tau= \pm \sqrt{\left(\sigma_{1}+\sigma_{3}\right) \frac{\sigma_{3}+\sigma_{1} \sigma_{3}^{\prime}}{1+\sigma_{3}^{\prime}}-\left(\frac{\sigma_{3}+\sigma_{1} \sigma_{3}^{\prime}}{1+\sigma_{3}^{\prime}}\right)^{2}-\sigma_{1} \sigma_{3}} .
$$

After simplification, this expression will take the form:

$$
\tau= \pm \frac{\sigma_{1}-\sigma_{3}}{1+\sigma_{3}^{\prime}} \sqrt{\sigma_{3}^{\prime}}
$$

Equations (17) and (18) represent the equation of the envelope line in parametric form. Let's find the slope coefficient of the tangent to the envelope line. To do this, we differentiate equation (16) with respect to the argument $\sigma$, considering the principal stresses $\sigma_{1}, \sigma_{3}$ as constant values:

$$
2 \sigma+2 \tau \tau^{\prime}-\left(\sigma_{1}-\sigma_{3}\right)=0
$$

From here:

$$
\tau^{\prime}=\frac{\sigma_{1}+\sigma_{3}-2 \sigma}{2 \tau}
$$

Here $\tau^{\prime}$ is the slope coefficient of the tangent lines to the limit circle. Introducing the envelope line equation (17), (18) into this expression gives:

$$
\tau^{\prime}=\frac{1-\sigma_{3}^{\prime}}{2 \sqrt{\sigma_{3}^{\prime}}}
$$

In this expression $\tau^{\prime}$ is already the slope coefficient of the tangents to the envelope line of Mohr's limit circles. At the point of intersection of the envelope line with the axis of normal stress $\sigma$, the slope coefficient of the tangent is equal to infinity:

$$
\tau_{\tau=0}^{\prime}=\infty
$$

The slope coefficient of the tangent to the envelope line at $\sigma \rightarrow \infty$ is equal to zero. In this area, the envelope line of the limit circles becomes parallel to the axis $\sigma$ Thus, the range of variation of the slope coefficient value of the tangent to the envelope line has the form: 


$$
\infty \leq \tau^{\prime} \leq 0
$$

Let's solve equation (19) with respect to $\sigma_{3}^{\prime}$ :

$$
\sigma_{3}^{\prime}=1+2 \tau^{\prime 2}-2 \tau^{\prime} \sqrt{1+\tau^{\prime 2}}
$$

From this expression, we determine the limits of change $\sigma_{3}^{\prime}: \sigma_{3}^{\prime}=0$ at $\tau^{\prime}=\infty$ and $\sigma_{3}^{\prime}$ $=1$ at $\tau^{\prime}=0$. Thus, the area of change $\sigma_{3}^{\prime}$ looks like:

$$
0 \leq \sigma_{3}^{\prime} \leq 1
$$

Let's assume that it was possible to exclude the parameter $\sigma_{1}$ from the equations (17) and (18), as well as the functions $\sigma_{3}\left(\sigma_{1}\right), \sigma_{3}^{\prime}\left(\sigma_{1}\right)$ determined by this parameter. This would lead to an explicit envelope equation

$$
\tau=\tau(\sigma)
$$

Substitute (22) into equation (16):

$$
\sigma^{2}+\tau(\sigma)^{2}-\left(\sigma_{1}+\sigma_{3}\right) \sigma+\sigma_{1} \sigma_{3}=0
$$

Equation (23) can now be viewed as a relationship between principal stresses $\sigma_{1}, \sigma_{3}$ that contains a parameter $\sigma$. As follows from the expression (23), this function is an algebraic equation of the second degree with respect to variables $\sigma_{1}, \sigma_{3}$, and has an asymptote, the slope coefficient of which is defined above - the dependence (21).

Of the lines defined by such equations, only the hyperbola has an asymptote (Korn, 1968). This is the basis for the search for the relationship between the principal stresses

$$
\sigma_{3}=\sigma_{3}\left(\sigma_{1}\right)
$$

in the form of a hyperbole. We represent the hyperbola equation in the form:

$$
\sqrt{\sigma_{1}^{2}+\left(\sigma_{3}-b\right)^{2}}-\sqrt{\sigma_{1}^{2}+\left(\sigma_{3}-a\right)^{2}}=c,
$$

where $a, b, c$-are the constants, $\sigma_{1}, \sigma_{3}$ - coordinates of an arbitrary point $\mathrm{M}$ of the hyperbola. 


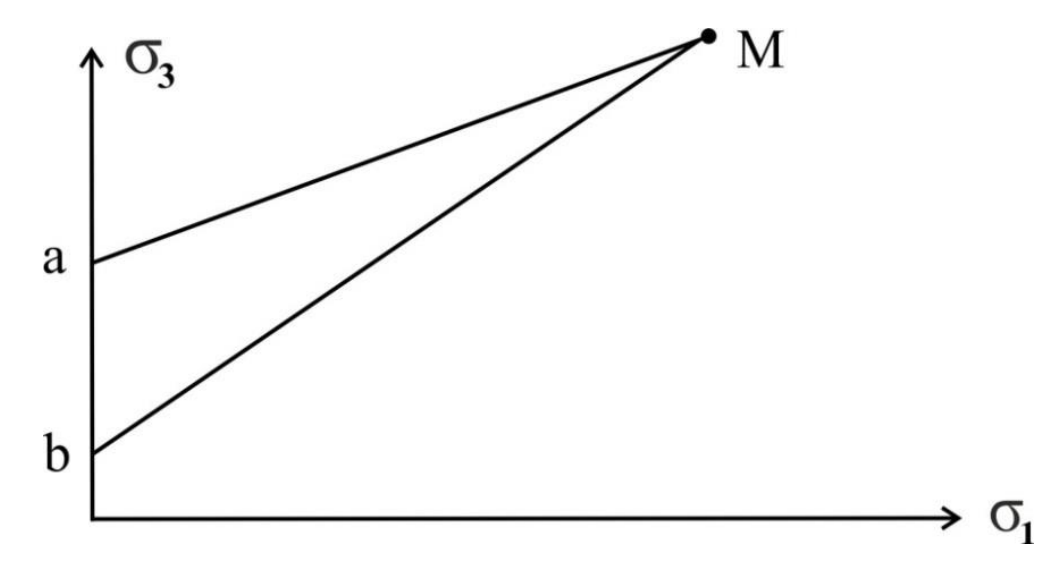

Fig. 4. To the derivation of the equation between the biggest and the smallest principal stresses of the Mohr limit circles

Solving this equation with respect to $\sigma_{3}$, we get:

$$
\sigma_{3}=\frac{a+b}{2}+\sqrt{\frac{c^{2}}{(a-b)^{2}-c^{2}} \sigma_{1}^{2}+\frac{c^{2}}{4}}
$$

For the curve (25) to have an asymptote, a limit $\lim _{\sigma_{1} \rightarrow \infty} \frac{\sigma_{3}}{\sigma_{1}}$ must exist. This limit exists and it is equal to:

$$
\lim _{\sigma_{1} \rightarrow \infty} \frac{\sigma_{3}}{\sigma_{1}}=\sqrt{\frac{c^{2}}{\left(a^{2}-b^{2}\right)-c^{2}}} .
$$

In addition, the value of this limit is equal to the slope coefficient of the asymptote, which was found above (15). In this way:

$$
\sqrt{\frac{c^{2}}{(a-b)^{2}-c}}=1, \text { or } c^{2}=\frac{(a-b)^{2}}{2} .
$$

The hyperbola equation now has the form:

$$
\sigma_{3}=\frac{a+b}{2}+\sqrt{\frac{(a-b)^{2}}{8}+\sigma_{1}^{2}} .
$$

The values of the constants $a, b$ are expressed through the ultimate tensile and compressive strengths of the material. Under axial compression, the principal stresses $\sigma_{1}=\sigma_{c}, \sigma_{3}=0$, where $\sigma_{c}$ is the ultimate strength of the material under axial compression. By substituting these values of the principal stresses in the equation (26), we find: 


$$
\frac{a^{2}+6 a b+b^{2}}{8}=\sigma_{c}^{2}
$$

Under axial tension, the principal ultimate stresses $\sigma_{1}=0, \sigma_{3}=\sigma_{p}$, where $\sigma_{p}$ is the value of the ultimate strength of the material under axial tension. Let us introduce these values into the equation (26) and represent the latter in the form:

$$
-\frac{a+b}{2}+\sigma_{p}=\sqrt{\frac{(a-b)^{2}}{8}} .
$$

Squaring both sides of this equality, we find:

$$
\sigma_{p}^{2}-(a+b) \sigma_{p}+\frac{a^{2}+6 a b+b^{2}}{8}=0
$$

Taking into account (27), we write this equation in the form:

$$
\sigma_{p}^{2}-(a+b) \sigma_{p}+\sigma_{c}^{2}=0 \text {. }
$$

From here we find:

$$
a+b=\frac{\sigma_{c}^{2}+\sigma_{p}^{2}}{\sigma_{p}} .
$$

Solving equations (27), (28) with respect to $a$ and $b$, we find:

$$
\begin{aligned}
& a=\frac{\sigma_{c}^{2}+\sigma_{p}^{2}}{2 \sigma_{p}}+\frac{\sigma_{c}^{2}-\sigma_{p}^{2}}{\sqrt{2 \sigma_{p}^{2}}} \\
& b=\frac{\sigma_{c}^{2}+\sigma_{p}^{2}}{2 \sigma_{p}}-\frac{\sigma_{c}^{2}-\sigma_{p}^{2}}{\sqrt{2 \sigma_{p}^{2}}}
\end{aligned}
$$

Let us introduce the found values of constants $a$ and $b$ into equation (26):

$$
\sigma_{3}=\frac{\sigma_{c}^{2}+\sigma_{p}^{2}}{2 \sigma_{p}}+\sqrt{\left(\frac{\sigma_{c}^{2}-\sigma_{p}^{2}}{2 \sigma_{p}}\right)^{2}+\sigma_{1}^{2}} .
$$




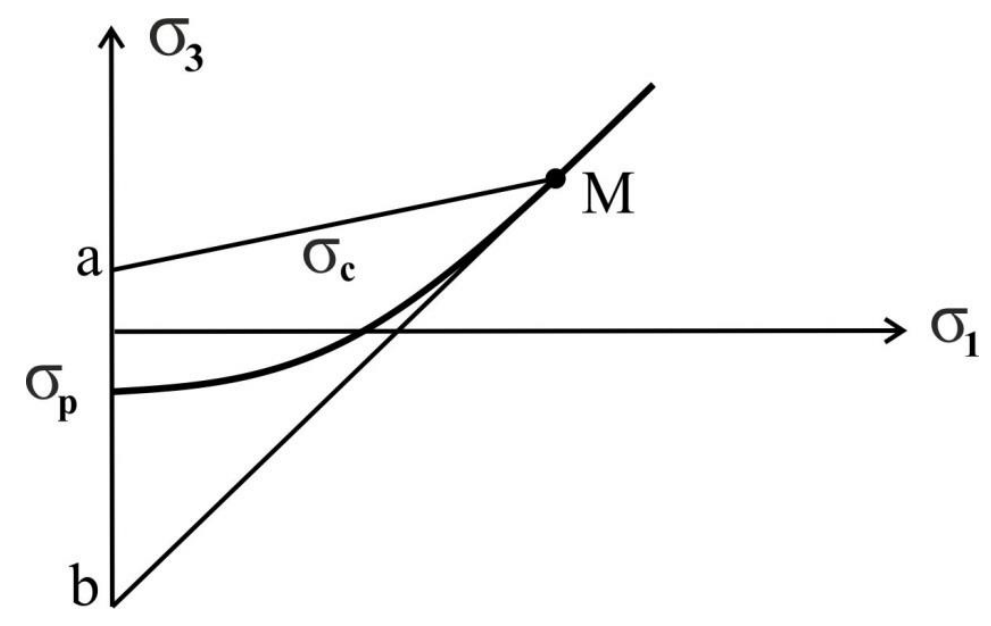

Fig. 5. Relationship between the principal stresses of the limiting states

The derivative of this function is equal to:

$$
\sigma_{3}^{\prime}=\frac{2 \sigma_{p} \sigma_{1}}{\sqrt{\left(\sigma_{c}^{2}-\sigma_{p}^{2}\right)^{2}+4 \sigma_{p}^{2} \sigma_{1}^{2}}}
$$

Let's write the equation of the envelope line of Mohr's limit circles in the final form:

$$
\sigma=\frac{\sigma_{3}+\sigma_{1} \sigma_{3}^{\prime}}{1+\sigma_{3}^{\prime}}, \tau= \pm \frac{\sigma_{1}-\sigma_{3}}{1+\sigma_{3}^{\prime}} \sqrt{\sigma_{3}^{\prime}}
$$

where $\sigma_{3}, \sigma_{3}$ are defined by the expressions (30), (31). If the compressive and tensile strengths of a material are established, then the equations (30), (31), (32) allow to determine all stress states that destroy this material and to construct an envelope line of Mohr's limit circles. As calculations show that the envelope equations found here, as well as the relationship between the limiting principal stresses, describe very well the results of testing materials in triaxial compression chambers.

\section{Conclusion}

None of the larger number of equations known to us, proposed as a quantitative description of Mohr's hypothesis, has a rigorous mathematical foundation. Our proposed equation:

$$
\sigma_{3}=\frac{a+b}{2}+\sqrt{\frac{(a-b)^{2}}{8}+\sigma_{1}^{2}}
$$


Duishenaliev, T.; Duishembiev, A.; Merkuryev, I. \& Ushanov, S.: Mathematical De...

differs in that it can be attributed to the category of grounded, mathematically derived equations. The equation expressing the quantitative relationship between the limiting values of the smallest $\sigma_{3}$ and the biggest $\sigma_{1}$ principal stresses is a very simple relationship. Nevertheless, it defines all those stress states that lead to the destruction of the material.

Using this equation, it is possible to determine the orientation of those areas along which the destruction occurs, to calculate the normal and shear stresses at these areas. Using this equation, you can construct the envelope line of the limit stress circles. The derived equation contains two constants. The way of their definition is indicated. If the values of these constants are established for a given material, then this equation can be used to determine all stress states that destroy this material.

\section{Acknowledgments}

The research was carried out within the framework of the project "Quantitative expression of Mohr's theory of strength" with the support of a grant from NRU "MPEI" for implementation of scientific research programs "Energy", "Electronics, Radio Engineering and IT", and "Industry 4.0, Technologies for Industry and Robotics in 2020-2022".

\section{References}

Duishenaliev, T. (2017). Non-classical solutions of the mechanics of a deformable body, MPEI Publishing House, ISBN 978-5-7046-1840-9, Moscow

Duishenaliev, T; Duishembiev, A. \& Mekenbaev, B. (2018). Criterion for verification of the generalized Law of Hooke. Chapter 01 in DAAAM International Scientific Book 2018, pp. 001-010, B. Katalinic (Ed.), Published by DAAAM International, ISBN 978-3-902734-19-8, ISSN 1726-9687, Vienna, Austria DOI: 10.2507/daaam.scibook.2018.09

Karman, T. (1915). Experiments on all-round compression. New ideas in technology. Digest of articles, No. 1, page numbers (51-102)

Koifman, M. and others. (1964). Strength of rocks in a volumetric stress state, Nauka, Moscow

Korn, G. (1968). Handbook of Mathematics, Nauka, Moscow, - 720 p.

Kuntysh, M. (1964). Research of the method for determining the main physical and mechanical characteristics of rocks used in solving rock pressure problems. Abstract of the dissertation of the candidate of technical sciences, Moscow, $-24 \mathrm{p}$.

Mohr, O. (1915). Causes the elastic limit and temporary resistance of materials. New ideas in technology: Collection 1. Education Publishing House, - 50 p.

Protodiakonov, M. (1962). Generalized equation to the limiting circles of Mohr stresses. Study of the physical and mechanical properties of rocks in relation to the problems of mountain pressure control: Collection of works, Publishing House of the USSR Academy of Sciences, Moscow, page numbers (13-18)

Stavrogin, A. (1961). Research of rocks in complex stress states, Mining journal, No. 3 , page numbers (34-39) 